\title{
Encorafenib, Binimetinib, and Cetuximab in BRAF V600E-Mutated Colorectal Cancer
}

\author{
S. Kopetz, A. Grothey, R. Yaeger, E. Van Cutsem, J. Desai, T. Yoshino, H. Wasan, \\ F. Ciardiello, F. Loupakis, Y.S. Hong, N. Steeghs, T.K. Guren, H.-T. Arkenau, \\ P. Garcia-Alfonso, P. Pfeiffer, S. Orlov, S. Lonardi, E. Elez, T.-W. Kim, \\ J.H.M. Schellens, C. Guo, A. Krishnan, J. Dekervel, V. Morris, A. Calvo Ferrandiz, \\ L.S. Tarpgaard, M. Braun, A. Gollerkeri, C. Keir, K. Maharry, M. Pickard, \\ J. Christy-Bittel, L. Anderson, V. Sandor, and J. Tabernero
}

ABSTRACT

The authors' full names, academic degrees, and affiliations are listed in the Appendix. Address reprint requests to Dr. Tabernero at Vall d'Hebron University Hospital, Vall d'Hebron Institute of Oncology (VHIO), Passeig de la Vall d'Hebron 119-129, 08035 Barcelona Spain, or at jtabernero@vhio.net.

A list of committee members and principal investigators in this trial is provided in the Supplementary Appendix, available at NEJM.org.

This article was published on September 30, 2019, at NEJM.org.

N Engl J Med 2019;381:1632-43. DOI: 10.1056/NEJMoa1908075

Copyright (c) 2019 Massachusetts Medical Society.

\section{BACKGROUND}

Patients with metastatic colorectal cancer with the BRAF V600E mutation have a poor prognosis, with a median overall survival of 4 to 6 months after failure of initial therapy. Inhibition of BRAF alone has limited activity because of pathway reactivation through epidermal growth factor receptor signaling.

\section{METHODS}

In this open-label, phase 3 trial, we enrolled 665 patients with BRAF V600Emutated metastatic colorectal cancer who had had disease progression after one or two previous regimens. Patients were randomly assigned in a 1:1:1 ratio to receive encorafenib, binimetinib, and cetuximab (triplet-therapy group); encorafenib and cetuximab (doublet-therapy group); or the investigators' choice of either cetuximab and irinotecan or cetuximab and FOLFIRI (folinic acid, fluorouracil, and irinotecan) (control group). The primary end points were overall survival and objective response rate in the triplet-therapy group as compared with the control group. A secondary end point was overall survival in the doublet-therapy group as compared with the control group. We report here the results of a prespecified interim analysis.

\section{RESULTS}

The median overall survival was 9.0 months in the triplet-therapy group and 5.4 months in the control group (hazard ratio for death, 0.52; 95\% confidence interval [CI], 0.39 to $0.70 ; \mathrm{P}<0.001)$. The confirmed response rate was $26 \%$ (95\% CI, 18 to 35 ) in the triplet-therapy group and $2 \%$ ( $95 \%$ CI, 0 to 7 ) in the control group $(\mathrm{P}<0.001)$. The median overall survival in the doublet-therapy group was 8.4 months (hazard ratio for death vs. control, $0.60 ; 95 \%$ CI, 0.45 to 0.79; $\mathrm{P}<0.001$ ). Adverse events of grade 3 or higher occurred in $58 \%$ of patients in the triplet-therapy group, in $50 \%$ in the doublet-therapy group, and in $61 \%$ in the control group.

CONCLUSIONS

A combination of encorafenib, cetuximab, and binimetinib resulted in significantly longer overall survival and a higher response rate than standard therapy in patients with metastatic colorectal cancer with the BRAF V600E mutation. (Funded by Array BioPharma and others; BEACON CRC ClinicalTrials.gov number, NCT02928224; EudraCT number, 2015-005805-35.) 
T HE BRAF VGOOE MUTATION OCCURS IN approximately $10 \%$ of patients with metastatic colorectal cancer, with recent estimates ranging from as low as $5 \%$ to as high as $21 \% .^{1-10}$ This mutation identifies a distinct subtype of colorectal cancer that has a poor prognosis. ${ }^{11}$ Initial standard chemotherapy for BRAF V600E-mutated colorectal cancer results in poor outcomes, and attempts to intensify therapy have met with limited success. ${ }^{12}$ After failure of initial therapy, subsequent lines of treatment have minimal effect, and rapidly progressive disease and short overall survival are seen. Recently reported results of a phase 2 trial of second-line and third-line therapy in this population showed an objective response rate of $4 \%$, progressionfree survival of 2 months, and overall survival of 5.9 months in the control group, which received standard therapy (irinotecan plus cetuximab). ${ }^{13}$

Although BRAF V600E is a driver mutation found in multiple tumor types, and BRAF inhibitors have clinical activity in BRAF V600Emutated melanoma and non-small-cell lung cancer, BRAF inhibitors alone have limited activity in BRAF V600E-mutated colorectal cancer. ${ }^{14-17}$ Preclinical models of BRAF V600E-mutated colorectal cancer have shown that BRAF inhibition causes rapid feedback activation through the epidermal growth factor receptor (EGFR); thus, BRAF inhibitor treatment alone does not sufficiently inhibit pathway signaling, which explains the lack of clinical efficacy of BRAF inhibition in this type of cancer. ${ }^{18-20}$ Translation of these findings into clinical trials has shown that BRAF inhibitors have increased antitumor activity in BRAF V600-mutated colorectal cancer when combined with anti-EGFR monoclonal antibodies. ${ }^{21-23}$ Preclinical studies have suggested that combined inhibition of BRAF and mitogen-activated protein kinase (MAPK) kinase (MEK) is more effective than BRAF inhibitors combined with antiEGFR agents. This finding was validated clinically in subsequent phase 1 and phase 2 trials that combined BRAF inhibitors with both anti-EGFR monoclonal antibodies and MEK inhibitors. ${ }^{18,24,25}$

Encorafenib is a BRAF inhibitor with more prolonged pharmacodynamic activity than other approved BRAF inhibitors. ${ }^{26}$ The combination of the BRAF inhibitor encorafenib and the antiEGFR monoclonal antibody cetuximab showed promising activity in early-phase clinical trials when compared with reported outcomes with standard treatment options and outcomes from early-phase trials of triplet regimens that combined an anti-EGFR antibody with other BRAF inhibitors plus either an MEK inhibitor or irinotecan. $^{13,21,27}$ We therefore sought to evaluate whether treatment with the combination of encorafenib plus cetuximab with or without the MEK inhibitor binimetinib would result in longer overall survival than standard therapy in patients with BRAF V600E-mutated metastatic colorectal cancer who had had disease progression after one or two previous lines of therapy. Before initiation of the randomized phase of the trial, a group of patients was enrolled in a lead-in phase to evaluate the safety of the triplet regimen. ${ }^{28}$

\section{METHODS}

\section{TRIAL OVERSIGHT}

The trial was approved by the institutional review board or independent ethics committee at each center and was conducted in accordance with the requirements of the regulatory authorities of each country and with the provisions of the Declaration of Helsinki and the Good Clinical Practice guidelines of the International Council on Harmonisation. All patients provided written informed consent. The steering committee and one of the sponsors (Array BioPharma) jointly designed the trial and reviewed the data, with the participation of the authors. All the investigators collected the data. Six of the authors wrote the first draft of the manuscript, with professional medical writing assistance funded by Array BioPharma, and all the authors contributed to subsequent drafts. All the authors vouch for the accuracy and completeness of the data and analyses and for the fidelity of the trial to the protocol (available with the full text of this article at NEJM.org).

\section{TRIAL DESIGN, PATIENTS, AND TREATMENTS}

The BEACON CRC (Binimetinib, Encorafenib, and Cetuximab Combined to Treat BRAF-Mutant Colorectal Cancer) trial is a global, multicenter, randomized, open-label, phase 3 trial. We enrolled patients with histologically or cytologically confirmed metastatic colorectal cancer with the BRAF V600E mutation who had had disease progression after one or two previous treatment 
regimens. Additional inclusion and exclusion criteria are provided in the trial protocol.

We randomly assigned patients in a 1:1:1 ratio to one of three groups. Patients in the triplettherapy group received encorafenib (300 mg daily), binimetinib (45 mg twice daily), and cetuximab (400 mg per square meter of body-surface area as an initial dose, then $250 \mathrm{mg}$ per square meter weekly). Patients in the doublet-therapy group received encorafenib and cetuximab, administered in the same doses and on the same schedule as the triplet regimen. Patients in the control group received the investigators' choice of either cetuximab (administered in the same doses and on the same schedule as the other regimens) and irinotecan (180 mg per square meter on days 1 and 15) or cetuximab and FOLFIRI (folinic acid [180 mg per square meter, administered on days 1 and 15], fluorouracil [400 $\mathrm{mg}$ per square meter as an initial dose, then $1200 \mathrm{mg}$ per square meter per day for 2 days, initiated on days 1 and 15], and irinotecan [at the same dose and on the same schedule as the other regimens]). Randomization was stratified according to Eastern Cooperative Oncology Group (ECOG) performancestatus score $(0$ or 1 [scores range from 0 to 5 , with higher scores reflecting greater disability]), previous use of irinotecan (yes or no), and cetuximab formulation (U.S.-licensed or Europeanapproved formulation). Treatment was administered in 28-day cycles until disease progression, unacceptable toxic effects, withdrawal of consent, initiation of subsequent anticancer therapy, or death. Crossover was not permitted before the data cutoff date.

\section{END POINTS}

The original sole primary end point was overall survival in the triplet-therapy group as compared with the control group. The protocol was amended to include an additional primary end point of the objective response rate in the triplet-therapy group as compared with the control group, as assessed by independent central reviewers who were unaware of the treatment assignments, and the initial interim analysis of overall survival (the results of which are reported here) was added in an attempt to expeditiously assess efficacy. Secondary end points included overall survival in the doublet-therapy group as compared with the control group, as well as progression-free sur- vival, duration of response, and safety in all groups. Tumor assessments were performed according to Response Evaluation Criteria in Solid Tumors (RECIST), version 1.1, ${ }^{29}$ every 6 weeks from the date of randomization for the first 24 weeks of treatment, then every 12 weeks thereafter until disease progression, withdrawal of consent, initiation of subsequent anticancer therapy, loss to follow-up, or death, regardless of whether the trial treatment was discontinued. All responses were confirmed by means of subsequent imaging performed at least 4 weeks after the initial response. The central review of imaging data was performed retrospectively by readers who were unaware of the treatment assignments. The incidence and severity of adverse events were assessed according to the National Cancer Institute Common Terminology Criteria for Adverse Events, version 4.03. ${ }^{30}$

\section{STATISTICAL ANALYSIS}

The type I error rate for the primary end points was controlled with the use of a fallback procedure described by Wiens and Dmitrienko. ${ }^{31}$ The end point of objective response rate in the triplet-therapy group as compared with the control group was assigned a one-sided alpha level of 0.005 . The remaining 0.020 was assigned to the end point of overall survival in the triplet-therapy group as compared with the control group.

To incorporate testing of selected secondary end points, a gatekeeping procedure with hierarchical testing was also used to account for the multiple comparisons (see the Supplementary Appendix, available at NEJM.org). The trial was not powered to formally compare the results in the triplet-therapy group with those in the doublet-therapy group.

The sample size was driven by the secondary end point of overall survival in the doublet-therapy group as compared with the control group. For this comparison, we calculated that 338 deaths would be required to give the trial $90 \%$ power to detect a hazard ratio for death of 0.70 , with the use of a stratified log-rank test at a onesided significance level of 0.025 . The number of patients who would need to be included in the primary analysis of objective response rate in the triplet-therapy group as compared with the control group was based on an assumption that the objective response rate would be $10 \%$ in the 
control group and 30\% in the triplet-therapy group; we calculated that 110 patients per group would provide $88 \%$ power, at a one-sided significance level of 0.005 , to show the higher objective response rate in the triplet-therapy group. The end point was tested with the use of a CochranMantel-Haenszel test. The initial analysis was performed by an independent statistician and reviewed by the independent data monitoring committee, which recommended whether the sponsor would have access to unblinded data on the basis of planned boundaries for futility and superiority.

Unless otherwise stated, the time-to-event end points were assessed in all patients who underwent randomization (i.e., the intention-to-treat population). Objective response was assessed in the first 331 patients who underwent randomization. Safety was assessed on the basis of adverse events and laboratory abnormalities and was evaluated in patients who received at least one dose of any trial drug and had at least one post-treatment safety assessment. For the purposes of this summary, all reported $\mathrm{P}$ values are two-sided. Additional details regarding the trial design and analysis methods are provided in the Supplementary Appendix.

\section{RESULTS}

\section{PATIENTS}

A total of 1677 patients were screened for eligibility. From May 2017 through January 2019, a total of 665 patients underwent randomization: 224 patients were assigned to the triplet-therapy group, 220 patients to the doublet-therapy group, and 221 patients to the control group (Fig. S1 in the Supplementary Appendix). The characteristics of the patients at baseline were similar across the three groups (Table 1). Baseline characteristics of the 331 patients included in the primary analysis of objective response rate are described in Table S1.

\section{EFFICACY}

As of the data cutoff date for this prespecified interim analysis (February 11, 2019), the median duration of follow-up for survival was 7.8 months across the three groups. Kaplan-Meier curves for survival are shown in Figure 1. The median overall survival was 9.0 months $(95 \%$ confidence interval [CI], 8.0 to 11.4 ) in the triplet-therapy group and 5.4 months (95\% CI, 4.8 to 6.6) in the control group. The risk of death was significantly lower (by 48\%) in the triplet-therapy group than in the control group (hazard ratio, 0.52; 95\% CI, 0.39 to $0.70 ; \mathrm{P}<0.001)$. The results of subgroup analyses were consistent with those of the overall analysis (Fig. 1C). The median overall survival was 8.4 months (95\% CI, 7.5 to 11.0 ) in the doublet-therapy group, and the risk of death was significantly lower than that in the control group (hazard ratio, 0.60 ; 95\% CI, 0.45 to 0.79 ; $\mathrm{P}<0.001)$. In a descriptive analysis of survival comparing the triplet regimen with the doublet regimen, the estimated 6-month survival was $71 \%$ in the triplet-therapy group and $65 \%$ in the doublet-therapy group (hazard ratio for death, 0.79; 95\% CI, 0.59 to 1.06) (Fig. S2). In the control group, the estimated 6-month survival was $47 \%$.

The objective response rate was significantly higher in the triplet-therapy group than in the control group (Table 2 and Fig. 2). The independently reviewed confirmed objective response rate, assessed in the first 331 patients who underwent randomization, was 26\% (95\% CI, 18 to 35) in the triplet-therapy group and $2 \%$ (95\% CI, 0 to 7 ) in the control group $(\mathrm{P}<0.001)$. The objective response rate in the doublet-therapy group was $20 \%$ (95\% CI, 13 to 29), which was also significantly higher than that in the control group $(\mathrm{P}<0.001)$. A waterfall plot showing the best percentage change from baseline in the sum of the diameters of the target lesions in the triplettherapy group and the doublet-therapy group, as assessed by central review, indicates differences in the depth of response that favor the triplet regimen; the responses as assessed by local investigators were similar to those assessed by central review (Fig. S3 and Table S2). The influence of the number of lines of previous therapy on response rate was assessed on the basis of observations of higher response rates in earlier lines of therapy in phase 2 studies and observations in the safety lead-in phase. ${ }^{28,32,33}$ The response rate among patients with only one previous line of therapy was 34\% (95\% CI, 23 to 47) in the triplet-therapy group, 22\% (95\% CI, 14 to $33)$ in the doublet-therapy group, and $2 \%(95 \%$ CI, 0 to 9) in the control group.

Progression-free survival (as assessed by central review) was significantly longer in both the 


\begin{tabular}{|c|c|c|c|}
\hline Characteristic & $\begin{array}{l}\text { Triplet Regimen } \\
\qquad(\mathrm{N}=224)\end{array}$ & $\begin{array}{l}\text { Doublet Regimen } \\
\qquad(\mathrm{N}=\mathbf{2 2 0})\end{array}$ & $\begin{array}{c}\text { Control } \\
(N=221)\end{array}$ \\
\hline \multicolumn{4}{|l|}{ Sex-no. (\%) } \\
\hline Male & $105(47)$ & $115(52)$ & $94(43)$ \\
\hline Female & $119(53)$ & $105(48)$ & $127(57)$ \\
\hline \multicolumn{4}{|l|}{ Age $-y r$} \\
\hline Median & 62 & 61 & 60 \\
\hline Range & $26-85$ & $30-91$ & $27-91$ \\
\hline \multicolumn{4}{|l|}{ ECOG performance-status score - no. (\%) $\dagger$} \\
\hline 0 & $116(52)$ & $112(51)$ & $108(49)$ \\
\hline 1 & $108(48)$ & $104(47)$ & $113(51)$ \\
\hline 2 & 0 & $4(2)$ & 0 \\
\hline \multicolumn{4}{|l|}{ Location of primary tumor — no. (\%) } \\
\hline Left side of the colon, including rectum & $79(35)$ & $83(38)$ & $68(31)$ \\
\hline Right side of the colon & $126(56)$ & $110(50)$ & $119(54)$ \\
\hline Both left and right side of the colon or unknown location & $19(8)$ & $27(12)$ & $34(15)$ \\
\hline Involvement of $\geq 3$ organs - no. (\%) & $110(49)$ & $103(47)$ & $98(44)$ \\
\hline Presence of liver metastases — no. (\%) & $144(64)$ & $134(61)$ & $128(58)$ \\
\hline \multicolumn{4}{|l|}{ Primary tumor removed — no. (\%) } \\
\hline Completely resected & $133(59)$ & $123(56)$ & $122(55)$ \\
\hline Partially resected or unresected & $91(41)$ & $97(44)$ & $99(45)$ \\
\hline \multicolumn{4}{|l|}{ Previous lines of therapy — no. (\%) } \\
\hline 1 & $146(65)$ & $146(66)$ & $145(66)$ \\
\hline $2 \div$ & $78(35)$ & $74(34)$ & $76(34)$ \\
\hline High microsatellite instability — no. (\%)』 & $22(10)$ & $19(9)$ & $12(5)$ \\
\hline Baseline carcinoembryonic antigen level >5 $\mu \mathrm{g} /$ liter - no. (\%) & $179(80)$ & $153(70)$ & $178(81)$ \\
\hline Baseline C-reactive protein level >10 mg/liter — no. (\%) & $95(42)$ & $79(36)$ & $90(41)$ \\
\hline
\end{tabular}

* Percentages may not total 100 because of rounding. The triplet-therapy group received encorafenib, binimetinib, and cetuximab; the doublet-therapy group received encorafenib and cetuximab; and the control group received the investigators' choice of either cetuximab and irinotecan or cetuximab and FOLFIRI (folinic acid, fluorouracil, and irinotecan). $\dagger$ Eastern Cooperative Oncology Group (ECOG) performance-status scores range from 0 to 5 , with higher scores indicating greater disability.

$\$$ This category includes one patient in the triplet-therapy group and one patient in the control group who received more than two previous lines of therapy.

$\int$ High microsatellite instability was determined by polymerase chain reaction.

triplet-therapy group and the doublet-therapy group than in the control group. The median progression-free survival was 4.3 months (95\% CI, 4.1 to 5.2 ) in the triplet-therapy group, 4.2 months (95\% CI, 3.7 to 5.4 ) in the doublet-therapy group, and 1.5 months (95\% CI, 1.5 to 1.7 ) in the control group (Fig. S4). The hazard ratio for disease progression or death was 0.38 (95\% CI, 0.29 to 0.49 ) in the triplet-therapy group as compared with the control group $(\mathrm{P}<0.001)$ and 0.40 ( $95 \%$ CI, 0.31 to 0.52 ) in the doublet-therapy
Figure 1 (facing page). Overall Survival.

Panel A shows the Kaplan-Meier analysis of the probability of survival in the triplet-therapy group as compared with the control group, and Panel B the probability of survival in the doublet-therapy group as compared with the control group. Panel $\mathrm{C}$ shows the results of the subgroup analysis of overall survival in the triplettherapy group as compared with the control group. Eastern Cooperative Oncology Group (ECOG) performance-status scores range from 0 to 5 , with higher scores indicating greater disability. 


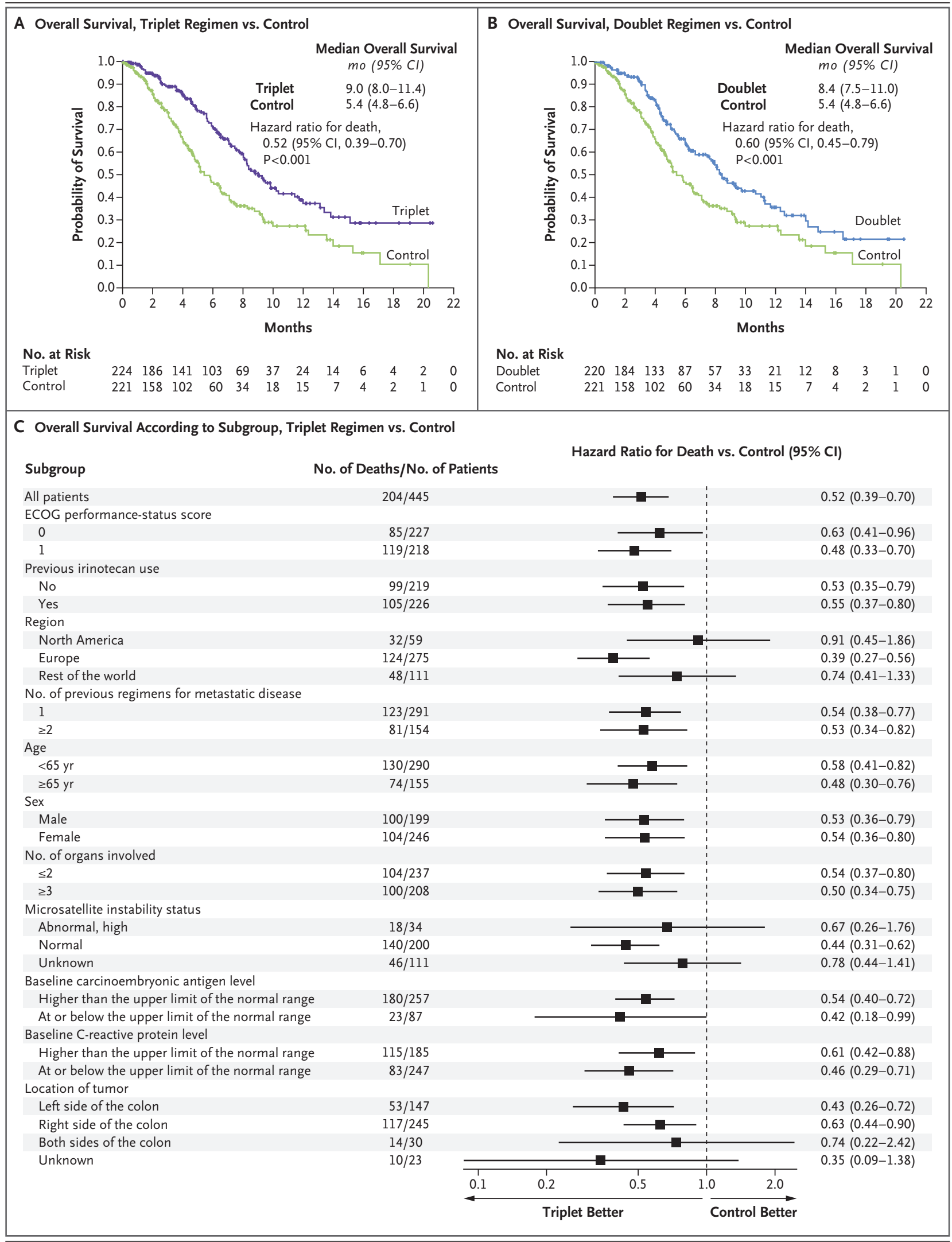

The New England Journal of Medicine 
Table 2. Tumor Response in Patients with Metastatic Colorectal Cancer with the BRAF V600E Mutation.*

\begin{tabular}{|c|c|c|c|}
\hline Variable & $\begin{array}{c}\text { Triplet } \\
\text { Regimen } \\
(\mathrm{N}=111)\end{array}$ & $\begin{array}{l}\text { Doublet } \\
\text { Regimen } \\
(\mathrm{N}=113)\end{array}$ & $\begin{array}{l}\text { Control } \\
(\mathrm{N}=107)\end{array}$ \\
\hline \multicolumn{4}{|l|}{ Objective response } \\
\hline Patients with a complete or partial response - no. (\%) & $29(26)$ & $23(20)$ & $2(2)$ \\
\hline $95 \% \mathrm{Cl}$ & $18-35$ & $13-29$ & $<1-7$ \\
\hline$P$ value vs. control & $<0.001$ & $<0.001$ & \\
\hline \multicolumn{4}{|l|}{ Best overall response - no. (\%) } \\
\hline Complete response & $4(4)$ & $6(5)$ & 0 \\
\hline Partial response & $25(23)$ & $17(15)$ & $2(2)$ \\
\hline Stable disease $†$ & $47(42)$ & $61(54)$ & $31(29)$ \\
\hline Progressive disease & $11(10)$ & $8(7)$ & $36(34)$ \\
\hline Could not be evaluated according to RECIST & $24(22)$ & $21(19)$ & $38(36)$ \\
\hline $\begin{array}{l}\text { Clinical progression or discontinuation because } \\
\text { of adverse event } \int\end{array}$ & $15(14)$ & $19(17)$ & $17(16)$ \\
\hline Insufficient data to assess response & $9(8)$ & $2(2)$ & $21(20)$ \\
\hline $\begin{array}{c}\text { Patients with duration of response } \geq 6 \mathrm{mo}-\text { no./total no. } \\
\text { of patients with a response (\%) }\end{array}$ & $7 / 29(24)$ & $10 / 23(43)$ & $1 / 2(50)$ \\
\hline $\begin{array}{l}\text { Patients with ongoing response and }<6 \text { mo follow-up } \\
\qquad- \text { no./total no. of patients with a response (\%) }\end{array}$ & $4 / 29$ (14) & $1 / 23(4)$ & 0 \\
\hline
\end{tabular}

* All responses were confirmed and were assessed by blinded independent central review according to Response Evaluation Criteria in Solid Tumors (RECIST), version 1.1. The first 331 patients who underwent randomization were included in the assessment of tumor response. Percentages may not total 100 because of rounding.

$\dagger$ This category refers to patients with measurable disease who had stable disease and patients with nonmeasurable disease who did not have a complete response or who did not have progressive disease according to RECIST.

$\uparrow$ This category includes 3 patients in the triplet-therapy group who had a confirmed partial response as determined by local assessment (these patients underwent scanning at outside institutions at baseline, and the scans were not available for central assessment).

$\int$ This category refers to patients who discontinued the trial regimen because of adverse events or whose disease could not be assessed centrally but who had clinical or radiologic disease progression according to local assessment.

ๆ This category refers to patients who did not receive treatment, who withdrew consent, whose best response was stable disease within 42 days after randomization, who had no baseline scans available, or who had no postbaseline scans and no evidence of clinical progression or an adverse event.

group as compared with the control group $(\mathrm{P}<0.001)$. As of the data cutoff date, the median follow-up for progression-free survival was 5.4 months.

\section{SAFETY}

The most frequently occurring adverse events and laboratory abnormalities of any cause are summarized in Table 3. The most common adverse events in the triplet-therapy group were gastrointestinal-related and skin-related events, including diarrhea, nausea, vomiting, and acneiform dermatitis. Low hemoglobin level or anemia was a common laboratory abnormality. Table S3 lists selected adverse events that occurred during the trial, grouped according to clinically similar events that are commonly associated with BRAF and MEK inhibitors. Class-related toxic effects of MEK inhibitors, including serous retinopathy and left ventricular dysfunction, occurred at rates similar to those described previously ${ }^{15,16,28}$ and were managed with treatment interruptions with or without subsequent dose reduction. Adverse events of grade 3 or higher were observed in 58\% of patients in the triplet-therapy group, in 50\% in the doublet-therapy group, and in $61 \%$ in the control group. Discontinuation of therapy primarily because of an adverse event was seen in $7 \%$ of patients in the triplet-therapy group, in $8 \%$ in the doublet-therapy group, and in $11 \%$ in the 


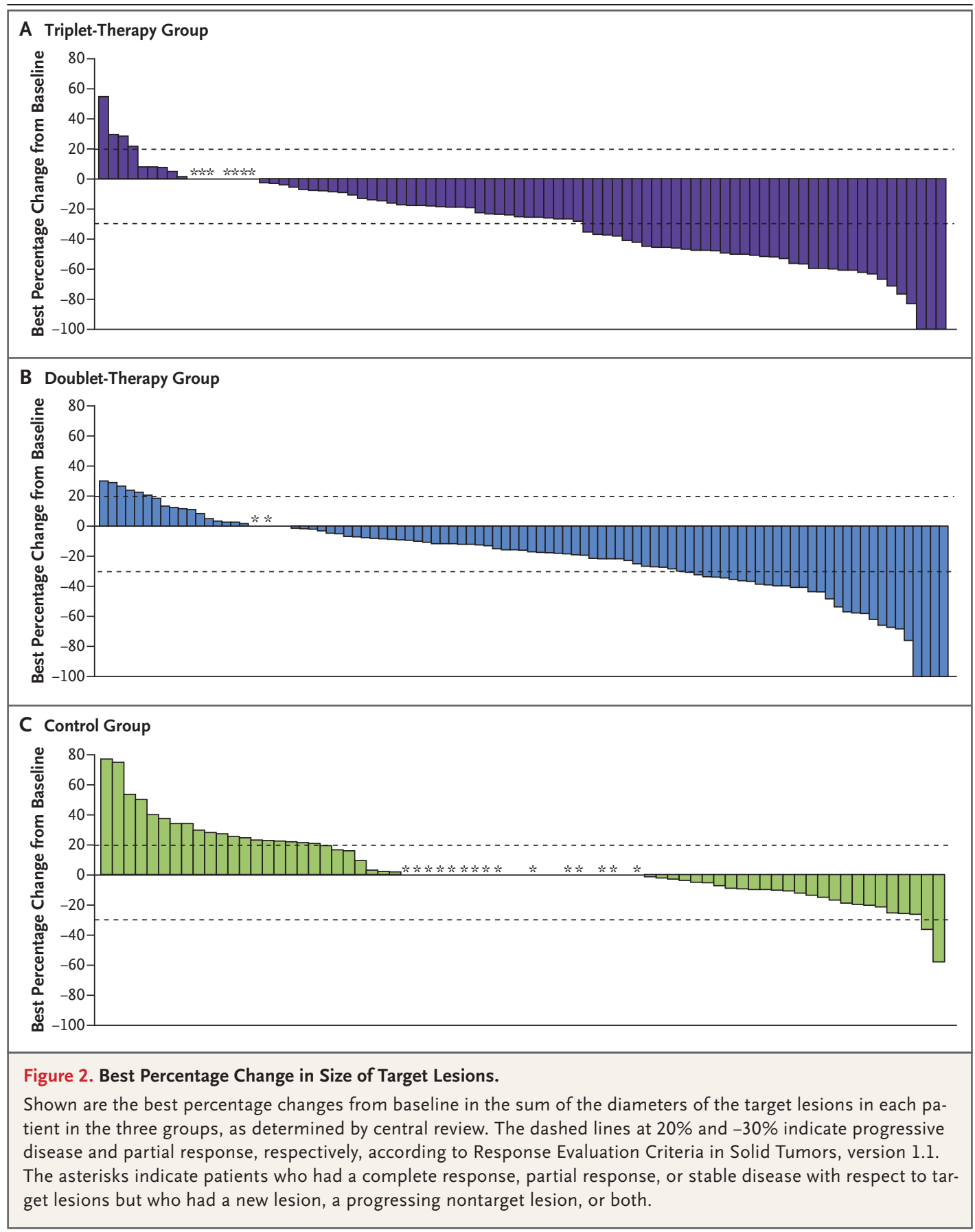

control group. Fatal adverse events occurred in $4 \%, 3 \%$, and $4 \%$ of the patients, respectively. Three of the deaths were determined by the investigators to be related to treatment: one death (in the triplet-therapy group) was from colonic perforation, one (in the control group) was from anaphylaxis, and one (in the control group) was from respiratory failure.

The median duration of exposure to trial drugs was 21 weeks in the triplet-therapy group, 19 weeks in the doublet-therapy group, and 7 weeks in the control group. The median relative dose 


\begin{tabular}{|c|c|c|c|c|c|c|}
\hline \multirow[t]{2}{*}{ Variable } & \multicolumn{2}{|c|}{$\begin{array}{l}\text { Triplet Regimen } \\
\quad(\mathrm{N}=222)\end{array}$} & \multicolumn{2}{|c|}{$\begin{array}{l}\text { Doublet Regimen } \\
\qquad(\mathrm{N}=216)\end{array}$} & \multicolumn{2}{|c|}{$\begin{array}{l}\text { Control } \\
(\mathrm{N}=193)\end{array}$} \\
\hline & Any Grade & Grade $\geq 3$ & $\begin{array}{l}\text { Any Grade } \\
\text { number of }\end{array}$ & $\begin{array}{c}\text { Grade } \geq 3 \\
\text { nts (percent) }\end{array}$ & Any Grade & Grade $\geq 3$ \\
\hline \multicolumn{7}{|l|}{ Adverse events } \\
\hline Any adverse event & $217(98)$ & $128(58)$ & $212(98)$ & $108(50)$ & $188(97)$ & $117(61)$ \\
\hline Diarrhea & $137(62)$ & $22(10)$ & $72(33)$ & $4(2)$ & $93(48)$ & $19(10)$ \\
\hline Acneiform dermatitis & $108(49)$ & $5(2)$ & $63(29)$ & $1(<1)$ & $76(39)$ & $5(3)$ \\
\hline Nausea & $100(45)$ & $10(5)$ & $74(34)$ & $1(<1)$ & $80(41)$ & $2(1)$ \\
\hline Vomiting & $85(38)$ & $9(4)$ & $46(21)$ & $3(1)$ & $56(29)$ & $5(3)$ \\
\hline Fatigue & $73(33)$ & $5(2)$ & $65(30)$ & $9(4)$ & $53(27)$ & $8(4)$ \\
\hline Abdominal pain & $65(29)$ & $13(6)$ & $49(23)$ & $5(2)$ & $48(25)$ & $9(5)$ \\
\hline Decreased appetite & $63(28)$ & $4(2)$ & $58(27)$ & $3(1)$ & $52(27)$ & $6(3)$ \\
\hline Asthenia & $55(25)$ & $7(3)$ & $46(21)$ & $7(3)$ & $49(25)$ & $9(5)$ \\
\hline Constipation & $55(25)$ & 0 & $33(15)$ & 0 & $35(18)$ & $2(1)$ \\
\hline Dry skin & $46(21)$ & $2(1)$ & $24(11)$ & 0 & $13(7)$ & $1(1)$ \\
\hline Pyrexia & $45(20)$ & $4(2)$ & $35(16)$ & $2(1)$ & $27(14)$ & $1(1)$ \\
\hline Rash & $42(19)$ & $1(<1)$ & $25(12)$ & 0 & $27(14)$ & $3(2)$ \\
\hline Stomatitis & $31(14)$ & $1(<1)$ & $12(6)$ & 0 & $44(23)$ & $4(2)$ \\
\hline $\begin{array}{l}\text { Palmar-plantar erythrodysesthesia } \\
\text { syndrome }\end{array}$ & $28(13)$ & 0 & $9(4)$ & $1(<1)$ & $14(7)$ & 0 \\
\hline Pruritus & $28(13)$ & 0 & $20(9)$ & 0 & $9(5)$ & 0 \\
\hline Back pain & $25(11)$ & $2(1)$ & $22(10)$ & $2(1)$ & $23(12)$ & $2(1)$ \\
\hline Blurred vision & $25(11)$ & 0 & $8(4)$ & 0 & $1(1)$ & 0 \\
\hline Peripheral edema & $24(11)$ & $1(<1)$ & $18(8)$ & 0 & $13(7)$ & $1(1)$ \\
\hline Weight decreased & $24(11)$ & $1(<1)$ & $21(10)$ & $1(<1)$ & $11(6)$ & 0 \\
\hline Arthralgia & $23(10)$ & 0 & 41 (19) & $2(1)$ & $1(1)$ & 0 \\
\hline Cough & $23(10)$ & 0 & $16(7)$ & $1(<1)$ & $10(5)$ & 0 \\
\hline Myalgia & $18(8)$ & 0 & $29(13)$ & $1(<1)$ & $4(2)$ & 0 \\
\hline Dyspnea & $17(8)$ & $2(1)$ & $23(11)$ & $2(1)$ & $17(9)$ & $5(3)$ \\
\hline Headache & $16(7)$ & 0 & $42(19)$ & 0 & $5(3)$ & 0 \\
\hline Pain in extremity & $15(7)$ & 0 & $22(10)$ & 0 & $1(1)$ & 0 \\
\hline Insomnia & $11(5)$ & 0 & $24(11)$ & 0 & $11(6)$ & 0 \\
\hline Musculoskeletal pain & $6(3)$ & 0 & $27(12)$ & 0 & $3(2)$ & 0 \\
\hline Melanocytic nevus & $1(<1)$ & 0 & $31(14)$ & 0 & 0 & 0 \\
\hline \multicolumn{7}{|l|}{ Abnormal laboratory values } \\
\hline Alanine aminotransferase & $51(23)$ & $4(2)$ & $36(17)$ & 0 & $50(26)$ & $5(3)$ \\
\hline Aspartate aminotransferase & $50(23)$ & $4(2)$ & $31(14)$ & $3(1)$ & $38(20)$ & $3(2)$ \\
\hline Bilirubin & $12(5)$ & $5(2)$ & $16(7)$ & $5(2)$ & $16(8)$ & $6(3)$ \\
\hline Creatine kinase & $52(23)$ & $6(3)$ & $6(3)$ & 0 & $13(7)$ & 0 \\
\hline Creatinine & $166(75)$ & $10(5)$ & $109(50)$ & $5(2)$ & $65(34)$ & $2(1)$ \\
\hline Hemoglobin & $125(56)$ & $24(11)$ & $70(32)$ & $9(4)$ & $85(44)$ & $8(4)$ \\
\hline
\end{tabular}

* Shown are adverse events of any grade and selected laboratory abnormalities reported in more than $10 \%$ of patients and adverse events of grade 3 or higher reported in more than $2 \%$ of patients in the triplet-therapy group or the doublet-therapy group. Adverse events were graded according to the National Cancer Institute Common Terminology Criteria for Adverse Events, version 4.03, and were coded according to the preferred terms from the Medical Dictionary of Regulatory Activities, version 21.0. 
intensities were $91 \%$ in the triplet-therapy group and $98 \%$ in the doublet-therapy group for encorafenib; $87 \%$ in the triplet-therapy group for binimetinib; and $91 \%$ in the triplet-therapy group and $93 \%$ in the doublet-therapy group for cetuximab.

\section{DISCUSSION}

This initial analysis of the BEACON CRC trial shows that the triplet regimen of encorafenib, binimetinib, and cetuximab resulted in longer overall survival and a higher objective response rate than a control regimen of cetuximab plus the investigators' choice of irinotecan-based chemotherapy in patients with BRAF V600E-mutated metastatic colorectal cancer who had had disease progression after one or two previous regimens. The doublet regimen of encorafenib and cetuximab also resulted in significantly longer overall survival and a higher objective response rate than were seen in the control group. The results in the control group were as expected on the basis of recently reported prospective data in a similar population and several published retrospective analyses. ${ }^{1,12,13,32,34}$

The triplet regimen was designed to be a combination of agents that would provide the most effective inhibition of the MAPK pathway. Preclinical and clinical studies showed that the lack of efficacy of single-agent BRAF or dual BRAF and MEK inhibition in BRAF V600E-mutated colorectal cancer is related to EGFR-mediated adaptive feedback - a finding that led to the development of a combination of BRAF, MEK, and EGFR inhibition through a series of iterative studies. ${ }^{17-23,25}$ Analyses of mechanisms of resistance in several studies reiterated the dependency of BRAF V600E-mutated metastatic colorectal cancer on the MAPK pathway, which is composed most prominently of KRAS, NRAS, BRAF, and MEK..$^{17,21,22,35,36}$ Suppression of MAPK signaling with encorafenib, binimetinib, and cetuximab represented the logical therapeutic strategy to address this persistent dependency. Despite the documented activity of the triplet regimen, resistance ultimately develops in many patients, and further characterization of these mechanisms is needed to further improve outcomes.

The trial was not powered to compare the two experimental groups directly, and such a comparison is further limited by the interim nature of this analysis. The analysis of overall survival that compared the triplet regimen with the doublet regimen showed a hazard ratio for death that favored the triplet regimen $(0.79 ; 95 \%$ CI, 0.59 to 1.06). A waterfall plot of the best percentage change in the sum of the diameters of the target lesions also indicated differences in the depth of response that favored the triplet regimen (Fig. S3).

The rate of adverse events was similar in the triplet-therapy group and the doublet-therapy group, and the frequency of toxic effects of grade 3 or higher was slightly higher in the control group than in either targeted-therapy group. The median duration of exposure to trial treatment was 21 weeks in the triplet-therapy group, 19 weeks in the doublet-therapy group, and 7 weeks in the control group. The inclusion of binimetinib as part of the triplet regimen added some additional toxic effects that were associated with MEK inhibition. The rate of treatment discontinuation and the relative dose intensity were similar in the triplet-therapy group and the doublet-therapy group. The observed safety profiles were generally consistent with those previously reported for each regimen and with the known effects of MEK, BRAF, and EGFR inhibitors. Headache, musculoskeletal pain, arthralgia, and myalgia occurred more frequently in the doublet-therapy group than in the triplet-therapy group but did not generally result in discontinuation of either component of the regimen. The less frequent occurrence of these adverse effects in the triplet-therapy group is consistent with the ability of MEK inhibition to mitigate some toxic effects associated with BRAF inhibition. ${ }^{15,16}$

This initial analysis in patients with BRAF V600E-mutated metastatic colorectal cancer who had had disease progression after one or two previous regimens showed that a triplet regimen of encorafenib, binimetinib, and cetuximab or a doublet regimen of encorafenib and cetuximab, as compared with current standard therapy, resulted in a significant and clinically relevant benefit with respect to overall survival and objective response rate. The side-effect profiles of both combination regimens allowed maintenance of high dose intensity for the majority of patients and are consistent with the known profile of each agent. Further follow-up is needed to better define the relative benefits of the triplet and doublet regimens.

Supported by Array BioPharma, Merck (for sites outside North America), ONO Pharmaceutical, and Pierre Fabre. 
Dr. Kopetz reports receiving advisory board fees from Amal Therapeutics, Amgen, AstraZeneca, Bayer Healthcare, Boehringer Ingelheim, Boston Biomedical, Eli Lilly, EMD Serono, Holy Stone Healthcare, Karyopharm Therapeutics, Merck, Novartis, Roche, and Symphogen, grant support, paid to M.D. Anderson Cancer Center, from AstraZeneca, holding stock options in Navire Pharma, and receiving lecture fees from Pierre Fabre Pharmaceuticals; Dr. Grothey, receiving consulting fees, paid to his institution, from Array BioPharma, Bayer Healthcare, Boston Biomedical, Genentech, and Pierre Fabre Pharmaceuticals; Dr. Yaeger, receiving grant support, paid to her institution, from Array BioPharma, Boehringer Ingelheim, GlaxoSmithKline, and Novartis, and fees for serving on a steering committee and travel support from Array BioPharma; Dr. Van Cutsem, receiving grant support, paid to the University of Leuven, from Amgen and Boehringer Ingelheim, and advisory board fees from Array BioPharma, AstraZeneca, Bayer Healthcare, Bristol-Myers Squibb, Celgene, Daiichi Sankyo, Eli Lilly, F. Hoffmann-La Roche, Incyte Corporation, Ipsen Biopharmaceuticals, Merck, Novartis, Pierre Fabre Pharmaceuticals, and Sirtex Medical; Dr. Desai, receiving fees for institutional collaboration, paid to his institution, from AstraZeneca, Bristol-Myers Squibb, Genentech USA, and GlaxoSmithKline, and consulting fees from BeiGene; Dr. Yoshino, receiving grant support, paid to National Cancer Center Hospital East, from Chugai Pharmaceutical, Daiichi Sankyo, GlaxoSmithKline, MSD KK, Novartis Pharma, ONO Pharmaceutical, Parexel International, Sanofi, and Sumitomo Dainippon Pharma; Dr. Wasan, receiving fees for serving on an end-point review committee from Array BioPharma, travel support from Bristol-Myers Squibb, advisory board fees and fees for serving on a speaker's bureau from EMD Serono, F. HoffmannLa Roche, and Servier, advisory board fees from Erytech, Incyte Corporation, and Shire, and grant support, paid to his institution, and advisory board fees and fees for serving on a speaker's bureau from Sirtex Medical; Dr. Ciardiello, receiving grant support, paid to his institution, from Amgen, AstraZeneca, Bayer Healthcare, F. Hoffmann-La Roche, and Merck KGaA, and advisory board fees from Array BioPharma, Bayer Healthcare, F. Hoffmann-La Roche, Merck KGaA, Pfizer, and Pierre Fabre
Pharmaceuticals; Dr. Loupakis, receiving lecture fees from Amgen and F. Hoffmann-La Roche, and advisory fees from Bayer; Dr. Lonardi, receiving advisory board fees from Amgen, Eli Lilly, and Merck; Dr. Elez, receiving lecture fees and advisory fees from Amgen, Merck, Pierre Fabre Pharmaceuticals, Sanofi Aventis, and Servier, and advisory board fees from F. Hoffmann-La Roche; Dr. Schellens, receiving consulting fees from Debiopharm and being employed by and holding stock in Modra Pharmaceuticals; Dr. Dekervel, receiving lecture fees from Amgen, Bayer, Ipsen Biopharmaceuticals, and Merck, travel support from Ipsen Biopharmaceuticals, and consulting fees from Novartis; Dr. Morris, receiving consulting fees from Array BioPharma; Dr. Gollerkeri, being employed by Array BioPharma; Dr. Keir, being employed by Array BioPharma and Novartis Pharma; Dr. Maharry, being employed by Array BioPharma; Dr. Pickard, being employed by and holding stock in Array BioPharma; Dr. Christy-Bittel, being employed by Array BioPharma and holding stock in Gilead Sciences; Dr. Sandor, being employed by and holding stock in Array BioPharma; and Dr. Tabernero, receiving consulting fees from Array BioPharma, AstraZeneca, Bayer, BeiGene, Biocartis, Boehringer Ingelheim, Chugai Pharmaceutical, Eli Lilly and Company, F. Hoffmann-La Roche, Foundation Medicine, Genentech, Genmab, HalioDX SAS, Halozyme, Imugene, Inflection Biosciences, Ipsen Biosciences, Kura Oncology, Menarini Ricerche, Merck Serono, Merrimack Pharmaceuticals, Merus, Molecular Partners, MSD, Novartis, Peptomyc, Pfizer, Pharmacyclics (an AbbVie company), ProteoDesign, Rafael Pharmaceuticals, Roche Diagnostics, Sanofi, SeaGen, Seattle Genetics, Servier, Symphogen, Taiho Pharmaceutical, and VCN Biosciences. No other potential conflict of interest relevant to this article was reported.

Disclosure forms provided by the authors are available with the full text of this article at NEJM.org.

A data sharing statement provided by the authors is available with the full text of this article at NEJM.org.

We thank the patients and their families, as well as the participating trial teams, for making this trial possible and J.D. Cox, Ph.D. (Mayville Medical Communications), for medical writing and editorial assistance with an earlier version of the manuscript.

\section{APPENDIX}

The authors' full names and academic degrees are as follows: Scott Kopetz, M.D., Ph.D., Axel Grothey, M.D., Rona Yaeger, M.D., Eric Van Cutsem, M.D., Ph.D., Jayesh Desai, M.B., B.S., Takayuki Yoshino, M.D., Ph.D., Harpreet Wasan, M.D., Fortunato Ciardiello, M.D., Ph.D., Fotios Loupakis, M.D., Ph.D., Yong Sang Hong, M.D., Ph.D., Neeltje Steeghs, M.D., Ph.D., Tormod K. Guren, M.D., Ph.D., Hendrik-Tobias Arkenau, M.D., Ph.D., Pilar Garcia-Alfonso, M.D., Per Pfeiffer, M.D., Ph.D., Sergey Orlov, M.D., Ph.D., Sara Lonardi, M.D., Elena Elez, M.D., Ph.D., Tae-Won Kim, M.D., Ph.D., Jan H.M. Schellens, M.D., Ph.D., Christina Guo, M.B., B.S., Asha Krishnan, B.S., Jeroen Dekervel, M.D., Van Morris, M.D., Aitana Calvo Ferrandiz, M.D., Ph.D., L.S. Tarpgaard, M.D., Ph.D., Michael Braun, M.B., Ch.B., Ph.D., Ashwin Gollerkeri, M.D., Christopher Keir, M.D., Kati Maharry, Ph.D., Michael Pickard, Ph.D., Janna Christy-Bittel, M.S.N., Lisa Anderson, M.P.H., Victor Sandor, M.D., and Josep Tabernero, M.D., Ph.D.

The authors' affiliations are as follows: the University of Texas M.D. Anderson Cancer Center, Houston (S.K., V.M.); West Cancer Center and Research Institute, OneOncology, Germantown, TN (A. Grothey); Memorial Sloan Kettering Cancer Center, New York (R.Y., A.K.); University Hospital Gasthuisberg and University of Leuven, Leuven, Belgium (E.V.C., J. Dekervel); the Peter MacCallum Cancer Centre, Melbourne, VIC, Australia (J. Desai, C.G.); National Cancer Center Hospital East, Kashiwa, Japan (T.Y.); Hammersmith Hospital, Division of Cancer, Imperial College London (H.W.), and the Sarah Cannon Research Institute and University College London Cancer Institute (H.-T.A.), London, and the Christie NHS Foundation Trust/National Institute for Health Research Manchester Biomedical Research Centre, Manchester (M.B.) — all in the United Kingdom; the University of Campania Luigi Vanvitelli, Naples (F.C.), and Istituto Oncologico Veneto, Istituto di Ricovero e Cura a Carattere Scientifico, Padua (F.L., S.L.) - both in Italy; Asan Medical Center, University of Ulsan College of Medicine, Seoul, South Korea (Y.S.H., T.-W.K.); the Netherlands Cancer Institute, Amsterdam (N.S., J.H.M.S.); Oslo University Hospital, Oslo (T.K.G.); Hospital Gregorio Marañón, Madrid (P.G.-A., A.C.F.), and Vall d'Hebron University Hospital, Vall d'Hebron Institute of Oncology, UVic, IOB-Quiron, Barcelona (E.E., J.T.) — both in Spain; Odense University Hospital, Odense, Denmark (P.P., L.S.T.); Pavlov First St. Petersburg State Medical University, St. Petersburg, Russia (S.O.); and Array BioPharma, Boulder, CO (A. Gollerkeri, C.K., K.M., M.P., J.C.-B., L.A., V.S.). 


\section{REFERENCES}

1. Davies H, Bignell GR, Cox C, et al. Mutations of the BRAF gene in human cancer. Nature 2002;417:949-54.

2. De Roock W, Claes B, Bernasconi D, et al. Effects of KRAS, BRAF, NRAS, and PIK3CA mutations on the efficacy of cetuximab plus chemotherapy in chemo therapy-refractory metastatic colorecta cancer: a retrospective consortium analysis. Lancet Oncol 2010;11:753-62.

3. Sorbye H, Dragomir A, Sundström M et al. High BRAF mutation frequency and marked survival differences in subgroups according to KRAS/BRAF mutation status and tumor tissue availability in a prospec tive population-based metastatic colorectal cancer cohort. PLoS One 2015;10(6): e0131046.

4. Loupakis F, Ruzzo A, Cremolini C et al. KRAS codon 61, 146 and BRAF mutations predict resistance to cetuximab plus irinotecan in KRAS codon 12 and 13 wildtype metastatic colorectal cancer. Br J Cancer 2009;101:715-21.

5. AACR Project GENIE Consortium AACR Project GENIE: powering precision medicine through an international consortium. Cancer Discov 2017;7:818-31.

6. Cancer Genome Atlas Research Network. Comprehensive genomic character ization of squamous cell lung cancers. Nature 2012;489:519-25.

7. Yaeger R, Chatila WK, Lipsyc MD, et al. Clinical sequencing defines the genomic landscape of metastatic colorectal cancer. Cancer Cell 2018;33(1):125.e3-136.e3.

8. Barras D, Missiaglia E, Wirapati P, et al. BRAF V600E mutant colorectal cancer subtypes based on gene expression. Clin Cancer Res 2017;23:104-15.

9. Bylsma LC, Gillezeau C, Garawin T, et al. Prevalence of RAS and BRAF mutations in metastatic colorectal cancer (mCRC) patients by tumor location. J Clin Oncol 2018;36:Suppl:681. abstract.

10. Clarke CN, Kopetz ES. BRAF mutant colorectal cancer as a distinct subset of colorectal cancer: clinical characteristics, clinical behavior, and response to targeted therapies. J Gastrointest Oncol 2015;6: 660-7.

11. Tran B, Kopetz S, Tie J, et al. Impact of BRAF mutation and microsatellite in stability on the pattern of metastatic spread and prognosis in metastatic colorectal cancer. Cancer 2011;117:4623-32.

12. Loupakis F, Cremolini C, Masi G, et al. Initial therapy with FOLFOXIRI and bevacizumab for metastatic colorectal cancer. N Engl J Med 2014;371:1609-18.

13. Kopetz S, McDonough SL, Lenz H-J, et al. Randomized trial of irinotecan and cetuximab with or without vemurafenib in BRAF-mutant metastatic colorectal cancer (SWOG S1406). J Clin Oncol 2017;35: Suppl:3505. abstract.

14. Planchard D, Besse B, Groen HJM, et al. Dabrafenib plus trametinib in patients with previously treated BRAF(V600E)mutant metastatic non-small cell lung cancer: an open-label, multicentre phase 2 trial. Lancet Oncol 2016;17:984-93.

15. Dummer R, Ascierto PA, Gogas HJ, et al. Encorafenib plus binimetinib versus vemurafenib or encorafenib in patients with BRAF-mutant melanoma (COLUMBUS): a multicentre, open-label, randomised phase 3 trial. Lancet Oncol 2018;19:60315.

16. Dummer R, Ascierto PA, Gogas HJ, et al. Overall survival in patients with BRAF-mutant melanoma receiving encorafenib plus binimetinib versus vemurafenib or encorafenib (COLUMBUS): a multicentre, open-label, randomised, phase 3 trial. Lancet Oncol 2018;19:131527.

17. Kopetz S, Desai J, Chan E, et al. Phase II pilot study of vemurafenib in patients with metastatic BRAF-mutated colorectal cancer. J Clin Oncol 2015;33:4032-8.

18. Corcoran RB, Atreya CE, Falchook GS, et al. Combined BRAF and MEK inhibition with dabrafenib and trametinib in BRAF V600-mutant colorectal cancer. J Clin Oncol 2015;33:4023-31.

19. Prahallad A, Sun C, Huang S, et al. Unresponsiveness of colon cancer to BRAF(V600E) inhibition through feedback activation of EGFR. Nature 2012; 483:100-3.

20. Mao M, Tian F, Mariadason JM, et al. Resistance to BRAF inhibition in BRAFmutant colon cancer can be overcome with PI3K inhibition or demethylating agents. Clin Cancer Res 2013;19:65767.

21. Corcoran RB, André T, Atreya CE, et al. Combined BRAF, EGFR, and MEK inhibition in patients with $B R A F^{\mathrm{V} 600 \mathrm{E}}$-mutant colorectal cancer. Cancer Discov 2018;8: 428-43.

22. Hong DS, Morris VK, El Osta B, et al. Phase IB study of vemurafenib in combination with irinotecan and cetuximab in patients with metastatic colorectal cancer with BRAFV600E mutation. Cancer Discov 2016;6:1352-65.

23. Yaeger R, Cercek A, O'Reilly EM, et al. Pilot trial of combined BRAF and EGFR inhibition in BRAF-mutant metastatic colorectal cancer patients. Clin Cancer Res 2015;21:1313-20.

24. Corcoran RB, Dias-Santagata D, Bergethon K, Iafrate AJ, Settleman J, Engelman JA. BRAF gene amplification can promote acquired resistance to MEK inhibitors in cancer cells harboring the BRAF V600E mutation. Sci Signal 2010; 3(149):ra84.

25. Corcoran RB, Ebi H, Turke AB, et al. EGFR-mediated re-activation of MAPK signaling contributes to insensitivity of BRAF mutant colorectal cancers to RAF inhibition with vemurafenib. Cancer Discov 2012;2:227-35.

26. Delord JP, Robert C, Nyakas M, et al. Phase I dose-escalation and -expansion study of the braf inhibitor encorafenib (LGX818) in metastatic BRAF-mutant melanoma. Clin Cancer Res 2017;23:5339-48. 27. Tabernero J, Van Geel R, Guren TK, et al. Phase 2 results: encorafenib (ENCO) and cetuximab (CETUX) with or without alpelisib (ALP) in patients with advanced BRAF-mutant colorectal cancer (BRAFm CRC). J Clin Oncol 2016;34:Suppl:3544. abstract.

28. Van Cutsem E, Huijberts S, Grothey A, et al. Binimetinib, encorafenib, and cetuximab triplet therapy for patients with BRAF V600E-mutant metastatic colorectal cancer: safety lead-in results from the phase III BEACON colorectal cancer study. J Clin Oncol 2019;37:1460-9.

29. Eisenhauer EA, Therasse P, Bogaerts J, et al. New response evaluation criteria in solid tumours: revised RECIST guideline (version 1.1). Eur J Cancer 2009;45:228-47. 30. Department of Health and Human Services. Common terminology criteria for adverse events, version 4.0. (CTCAE). May 28, 2009 (https://evs.nci.nih.gov/ftp1/ CTCAE/CTCAE_4.03/Archive/CTCAE_4.0 _2009-05-29_QuickReference_8.5x11.pdf). 31. Wiens BL, Dmitrienko A. The fallback procedure for evaluating a single family of hypotheses. J Biopharm Stat 2005;15: 929-42.

32. Morris V, Overman MJ, Jiang Z-Q, et al. Progression-free survival remains poor over sequential lines of systemic therapy in patients with BRAF-mutated colorectal cancer. Clin Colorectal Cancer 2014;13: 164-71.

33. Chibaudel B, Bonnetain F, Shi Q, et al. Alternative end points to evaluate a therapeutic strategy in advanced colorectal cancer: evaluation of progression-free survival, duration of disease control, and time to failure of strategy - an Aide et Recherche en Cancerologie Digestive Group study. J Clin Oncol 2011;29:4199-204.

34. Peeters M, Price TJ, Cervantes A, et al. Final results from a randomized phase 3 study of FOLFIRI $\{+\mid-\}$ panitumumab for second-line treatment of metastatic colorectal cancer. Ann Oncol 2014;25:107-16. 35. Ahronian LG, Sennott EM, Van Allen $\mathrm{EM}$, et al. Clinical acquired resistance to RAF inhibitor combinations in BRAFmutant colorectal cancer through MAPK pathway alterations. Cancer Discov 2015; 5:358-67.

36. Yaeger R, Yao Z, Hyman DM, et al. Mechanisms of acquired resistance to BRAF V600E inhibition in colon cancers converge on RAF dimerization and are sensitive to its inhibition. Cancer Res 2017; 77:6513-23.

Copyright (c) 2019 Massachusetts Medical Society. 